\title{
Clinical outcomes of robotic mitral valve repair: a single-center experience in Korea
}

\author{
Ho Jin Kim, Joon Bum Kim, Sung-Ho Jung, Jae Won Lee \\ Department of Thoracic and Cardiovascular Surgery, Asan Medical Center, University of Ulsan College of Medicine, Seoul, Republic of Korea \\ Correspondence to: Jae Won Lee, MD, PhD. The Department of Thoracic and Cardiovascular Surgery, Asan Medical Center, University of Ulsan \\ College of Medicine, 88, Olympic-Ro 43-Gil, Songpa-Gu, Seoul 138-736, Republic of Korea. Email: jwlee@amc.seoul.kr.
}

\begin{abstract}
Background: Since the inception of robotic mitral valve repair (MV) in 2007 at our institution, it has become an acceptable surgical option with proven efficacy and safety. The objective of this study is to analyze the early and long-term clinical outcomes of patients undergoing robotic MV repair.

Methods: A total of 310 patients (aged 48.4 13.7 years, 201 males) undergoing robotic MV repair using the da Vinci system (Intuitive Surgical, Inc., Sunnyvale, CA) between August 2007 and December 2015 in our institution were evaluated. The preoperative demographics, operative profiles and postoperative outcomes including follow-up echocardiographic results were analyzed.

Results: Successful MV repair was achieved in 98.4\% (n=305) of patients, with no significant residual mitral regurgitation (MR) postoperatively. There were no early postoperative deaths. Early postoperative complications included: stroke $(n=3,1.0 \%)$, new onset dialysis $(n=1,0.3 \%)$ and reoperation $(n=3,1.0 \%)$. During a median follow-up of 55.7 months (inter-quartile range 30.3 to 81.3 months), six (1.9\%) patients died, while four patients underwent late reoperation for mitral regurgitation $(n=2)$ or infective endocarditis $(n=2)$. Major event-free survival at five years was $87.6 \%$. Late echocardiographic profiles ( $>6$ months) were obtained in 295 (95.2\%) patients. During follow-up, 32 (10.8\%) patients developed significant mitral regurgitation (MR > grade 2), while freedom from significant MR at five years was $86.5 \%$.

Conclusions: Robotic MV repair is a safe procedure with acceptable postoperative results, including low early postoperative morbidity and mortality and acceptable long-term repair durability.
\end{abstract}

Keywords: Mitral valve repair; robotic cardiac surgery; minimally invasive mitral valve surgery

Submitted Jul 28, 2016. Accepted for publication Oct 11, 2016.

doi: $10.21037 /$ acs.2016.10.02

View this article at: http://dx.doi.org/10.21037/acs.2016.10.02

\section{Introduction}

Robotic mitral valve (MV) repair has been demonstrated as a safe and acceptable procedure by dedicated robotic surgical teams worldwide $(1,2)$. Observational studies have demonstrated favorable short- and long-term clinical outcomes comparable to conventional median sternotomy or minimally invasive anterolateral thoracotomy (3). Consequently, robotic MV repair has become more prevalent over the last decade $(4,5)$.

Despite the recent encouraging clinical outcomes of robotic MV repair using the da Vinci Surgical System (Intuitive Surgical, Inc, Sunnyvale, California, USA), the employment of a robotic approach to $M V$ repair has been relatively slow in Asia (6). Such stagnation is largely due to concerns regarding its safety, efficacy, and costeffectiveness. In 2007, our institution initiated a roboticassisted cardiac surgery program, and has pioneered this cutting-edge field of cardiac surgery in Korea as well as the entirety of Asia. Since then, MV repair has been the most common robotic-assisted cardiac procedure performed in our institution. In this study, we present the early and longterm clinical outcomes of patients undergoing robotic MV repair and evaluate the postoperative complications and long-term results. 


\section{Methods}

\section{Patients}

From August 2007 to December 2015, 540 patients underwent robotic-assisted cardiac surgery using the da Vinci Surgical System at the Asan Medical Center, Seoul, Korea. Of these patients, 310 underwent MV repair and therefore constituted the study population. Concomitant robotic-assisted cardiac procedures were as follows: maze procedure in 65 patients, tricuspid valve repair in 43 and correction of congenital heart disease in 34 .

The operating surgeons with expertise in various approaches to the MV explained the risks and benefits of robotic MV repair; the decision to perform the robotic surgery was made, preferentially reflecting on the patient's wishes, after a thorough discussion. Those patients who required concomitant coronary artery surgery or aortic valve surgery; had significant peripheral arterial disease not amenable to peripheral cannulation; or had a chest wall deformity that hindered surgical access were not considered for a robotic procedure. The study was approved by the Asan Medical Center Ethics Committee/Review board. Due to the retrospective nature of this study, the requirement for patient informed consent was waived.

\section{Surgical procedures}

The standard operative techniques using the da Vinci Surgical System in our institution have been described in a previous study (6). Under conventional general anesthesia, the patient was intubated with a dual-lumen endotracheal tube, followed by insertion of a transesophageal echocardiography (TEE) probe and attachment of external defibrillator patches to the upper back. After cannulating the superior vena cava through the right internal jugular vein percutaneously by an experienced anesthesiologist, the patient was placed in a left semi-lateral decubitus position with the right side up at $30^{\circ}$ to the horizontal plane. Cardiopulmonary bypass (CPB) was established by cannulating the right femoral vein and artery; right femoral artery was always preferred for cannulation, but an alternative arterial cannulation to the axillary artery $(n=3)$ or direct transthoracic ascending aorta $(n=9)$ was performed in patients with severe atherosclerotic disease in the aorta or small femoral artery. Femoral cannulation was performed through a 3 - to 4-cm oblique infra-inguinal groin incision, exposing only the anterior side of the femoral vessels to avoid damage to the lymphatic channels.
A 4-cm mini-thoracotomy incision was made in the $4^{\text {th }}$ intercostal space anterior to the anterior axillary line (AAL), to be used as both a working and camera access port. Three robotic arm trocars were then inserted: the first one in the $3^{\text {rd }}$ intercostal space anterior to the AAL for the left arm, the second one in the $6^{\text {th }}$ intercostal space at the AAL for the right arm, and the third one in the $4^{\text {th }}$ intercostal space, two fingerbreadths medial to the mini-thoracotomy for the atrial retractor. After docking the da Vinci Surgical System, vacuumassisted CPB drainage was initiated to empty the heart. and being careful to preserve the phrenic nerve, a pericardiotomy was done. An antegrade cardioplegia catheter was inserted into the anterolateral surface of the ascending aorta using a 3-0 polypropylene suture with pledgets. A Chitwood transthoracic aortic clamp (Scanlan International, Minneapolis, MN, USA) or a Glauber aortic clamp (Cardiomedical GmbH, Langenhagen, Germany; distributed by the Sorin Group) was used to occlude the ascending aorta. Before August 2013, a Chitwood clamp was used in consecutive 219 patients, whereas a Glauber clamp was used thereafter $(n=91)$. After aortic cross-clamping (ACC), antegrade cold crystalloid cardioplegic solution (Custodiol HTK; Köhler Chemie $\mathrm{GmbH}$, Bensheim, Germany) was infused to achieve myocardial protection. A left atriotomy was performed through the interatrial groove and the MV was exposed using an atrial retractor. After MV repair, intraoperative TEE was performed by a cardiologist in the operating room to evaluate any residual mitral regurgitation (MR) which was graded as mild, moderate, or severe based on color Doppler flow images.

\section{Definitions and clinical follow-up}

The primary early outcomes of interest were early mortality (within 30 days of surgery) and major postoperative complications (reoperation for bleeding, acute renal failure requiring dialysis, low cardiac output syndrome requiring mechanical circulatory support, stroke, thoracotomy wound infection, groin wound infection, pneumonia). The primary long-term outcomes of interest were the all-cause mortality and major adverse cardiac events (MACE) [cerebrovascular accident (CVA), reoperation, operated valve infective endocarditis, and readmission due to cardiovascular events]. Other outcomes of interest were MV repair durability, assessed using echocardiography.

Clinical follow-up data were obtained every 3 to 6 months through visits to the outpatient clinic. Serial echocardiographic follow-up assessment was used to evaluate the long-term durability of $M V$ repair. 


\begin{tabular}{ll} 
Table 1 Baseline patient characteristics \\
\hline Characteristics \\
\hline Age, years & $48.4 \pm 13.7$ \\
Male gender & $201(64.8)$ \\
Body mass index, $\mathrm{kg} / \mathrm{m}^{2}$ & $24.3 \pm 3.8$ \\
Body surface area, $\mathrm{m}^{2}$ & $1.8 \pm 0.2$ \\
Comorbidities & \\
Hypertension & $98(31.6)$ \\
Diabetes mellitus & $23(7.4)$ \\
Chronic kidney disease & $3(1.0)$ \\
COPD & $5(1.6)$ \\
Atrial fibrillation & $74(23.9)$ \\
History of CVA & $10(3.2)$ \\
NYHA class 3 or 4 & $72(23.2)$ \\
Previous cardiac surgery & $3(1.0)$
\end{tabular}

Echocardiographic data

LV ejection fraction, \%

$63.7 \pm 6.7$

LV end-systolic dimension, $\mathrm{mm}$

$37.7 \pm 5.5$

$\mathrm{LV}$ end-diastolic dimension, $\mathrm{mm}$

$59.9 \pm 6.2$

LA diameter, $\mathrm{mm}$

$48.1 \pm 8.7$

Peak TR pressure gradient, $\mathrm{mmHg}$

$30.1 \pm 12.4$

$\mathrm{TR} \geq$ grade 3

$10(3.2)$

MR grade

Moderate-to-severe

23 (7.4)

Severe

287 (92.6)

\section{Etiology of MR}

Degenerative

Rheumatic

Infective endocarditis

$22(7.1)$

Congenital

4 (1.3)

MV prolapse involved

$\begin{array}{ll}\text { Anterior leaflet } & 66(21.3) \\ \text { Posterior leaflet } & 168(54.2) \\ \text { Both leaflet } & 76(24.5)\end{array}$

Values are $\mathrm{n}(\%)$, or mean \pm standard deviation, unless otherwise indicated. COPD, chronic obstructive pulmonary disease; CVA, cerebrovascular accident; NYHA, New York Heart Association; LV, left ventricular; LA, left atrium; TR, tricuspid regurgitation; $\mathrm{MR}$, mitral regurgitation, MV, mitral valve.

\section{Statistical analysis}

Categorical variables were presented as percentages and frequencies, and continuous variables were presented as either mean \pm standard deviation or median with range. KaplanMeier curves were constructed to delineate the MACE-free survival and freedom from significant postoperative MR. Pearson correlation analysis was performed to review ACC and CPB time by the chronological order of MVP to evaluate the learning period effect. All reported $\mathrm{P}$ values were twosided. R software, version 3.1.3 (http://www.r-project.org/) was used for statistical analysis.

\section{Results}

\section{Baseline characteristics and operative profiles}

The baseline demographic and clinical characteristics of all patients are summarized in Table 1. There were 201 $(64.8 \%)$ male patients with a mean age of 48.4 years. Out of these patients, 72 (23.2\%) were classified with New York Heart Association functional class 3 or 4 . Most patients undergoing robotic MV repair in our institution had low preoperative risk profiles. The most prevalent comorbidities were hypertension $(31.6 \%)$, atrial fibrillation $(23.9 \%)$ and diabetes mellitus (7.4\%). Three patients had a history of previous cardiac surgery using sternotomy $(n=2)$ or thoracotomy $(n=1)$. These procedures included ventricular septal defect closure and MV repair (via sternotomy) and patent ductus arteriosus closure (via thoracotomy). Degenerative MV pathology (84.8\%) was the most common etiology in the study cohort.

There was no conversion to sternotomy intraoperatively. The anterior MV leaflet was repaired in 142 (45.8\%) patients. Table 2 outlines a variety of repair techniques performed on patients using robotic assistance. Ring annuloplasty was performed in all patients with a MV annulus size greater than $26 \mathrm{~mm}$ (308 patients; 99.4\% patients). CPB and ACC times were $179.3 \pm 52.4$ and $104.8 \pm 36.2$ minutes, respectively. Maze procedure $(n=65,20.9 \%)$ and tricuspid annuloplasty $(n=43$, $13.8 \%$ ) were performed concomitantly during MV repair when appropriate.

\section{Early and long-term clinical outcomes}

There was no early mortality, and early major morbidity occurred in 25 of $310(8.0 \%)$ patients: low cardiac output syndrome in one $(0.3 \%)$ patient, stroke in three $(1.0 \%)$, bleeding requiring exploration in $11(3.5 \%)$, new onset 


\begin{tabular}{|c|c|}
\hline \multicolumn{2}{|l|}{ Variable } \\
\hline CPB time (minutes) & $179.3 \pm 52.4$ \\
\hline ACC time (minutes) & $104.8 \pm 36.2$ \\
\hline \multicolumn{2}{|l|}{ Mitral valve procedures } \\
\hline Ring annuloplasty & $308(99.4)$ \\
\hline Quadrangular/triangular resection & $151(48.7)$ \\
\hline Neo chordae formation & $115(37.1)$ \\
\hline Commissuroplasty & $94(30.3)$ \\
\hline Sliding annuloplasty & $18(5.8)$ \\
\hline Cleft repair & $9(2.9)$ \\
\hline Chordae transfer & $7(2.3)$ \\
\hline Chordae release & $3(1.0)$ \\
\hline Leaflet augmentation & $4(1.3)$ \\
\hline Papillary muscle repositioning & $2(0.6)$ \\
\hline \multicolumn{2}{|l|}{ Concomitant procedures } \\
\hline Maze procedure & $65(20.9)$ \\
\hline TAP & 43 (13.8) \\
\hline ASD/PFO closure & $34(11.0)$ \\
\hline LA reduction & $20(6.5)$ \\
\hline LA appendage resection & $3(1.0)$ \\
\hline \multicolumn{2}{|c|}{$\begin{array}{l}\text { Values are } \mathrm{n}(\%) \text {, or mean } \pm \text { standard deviation, unless otherwise } \\
\text { indicated. CPB, cardiopulmonary bypass; ACC, aortic cross- } \\
\text { clamping; TAP, tricuspid annuloplasty; ASD, atrial septal defect; } \\
\text { PFO, patent foramen ovale; LA, left atrium. }\end{array}$} \\
\hline
\end{tabular}

dialysis in one $(0.3 \%)$, wound infection in one $(0.3 \%)$, groin wound lymphocele in two $(0.6 \%)$ and prolonged ventilation greater than 24 hours in 15 (4.8\%). Median hospital stay was six days [inter-quartile range (IQR), 5 to 8 days]. Three hundred and five patients (98.4\%) demonstrated a successful MV repair with no or mild residual MR on postoperative echocardiographic assessment before hospital discharge. Reoperation within 30 days of surgery occurred in three $(1.0 \%)$ patients: The first patient required redo $\mathrm{MV}$ repair on postoperative day 26 due to exacerbated eccentric MR with hemolysis. The second patient required mitral valve replacement on postoperative day 12 due to the development of systolic anterior motion of the MV combined with severe left ventricular outflow tract obstruction, which was discovered via postoperative

\begin{tabular}{|c|c|}
\hline \multicolumn{2}{|l|}{ Clinical outcomes } \\
\hline \multicolumn{2}{|l|}{ Early outcomes } \\
\hline Early mortality (<30 days) & $0(0.0)$ \\
\hline \multicolumn{2}{|l|}{ Early major morbidity } \\
\hline LCOS requiring MCS & $1(0.3)$ \\
\hline Early CVA & $3(1.0)$ \\
\hline Bleeding & $11(3.5)$ \\
\hline New-onset dialysis & $1(0.3)$ \\
\hline Thoracotomy wound infection & $1(0.3)$ \\
\hline Pneumonia & $1(0.3)$ \\
\hline Prolonged ventilation (>24 hours) & $15(4.8)$ \\
\hline Groin wound lymphocele & $2(0.6)$ \\
\hline Reoperation (within 30 days) & $3(1.0)$ \\
\hline MV surgery & $2(0.6)$ \\
\hline Aorta replacement due to dissection & $1(0.3)$ \\
\hline Postoperative ICU stay, days (IQR) & $1[1-2]$ \\
\hline Postoperative hospital stay, days (IQR) & $6[5-8]$ \\
\hline \multicolumn{2}{|l|}{ Long-term outcomes } \\
\hline All-cause mortality & $6(1.9)$ \\
\hline MACE & $36(11.6)$ \\
\hline Redo MV surgery & $2(0.6)$ \\
\hline Revascularization & $2(0.6)$ \\
\hline Late CVA & $4(1.3)$ \\
\hline Infective endocarditis & $2(0.6)$ \\
\hline Hospitalization due to cardiac cause & $22(7.1)$ \\
\hline Composite outcome (death + MACE) & $42(13.5)$ \\
\hline
\end{tabular}

Values are $n(\%)$, or mean \pm standard deviation, unless otherwise indicated. LCOS, low cardiac output syndrome; MCS, mechanical circulatory support; CVA, cerebrovascular accident; ICU, intensive care unit; IQR, inter-quartile range; MACE, major cardiac adverse event.

echocardiographic assessment. The last patient underwent ascending aorta and hemi-arch replacement on postoperative day three due to aortic dissection originating from the root catheter insertion site (Table 3).

During a median follow-up of 55.7 months (IQR, 30.3 to 81.3 months), late mortality occurred in $6(1.9 \%)$ patients. Major adverse cardiac outcomes (MACE) included late 


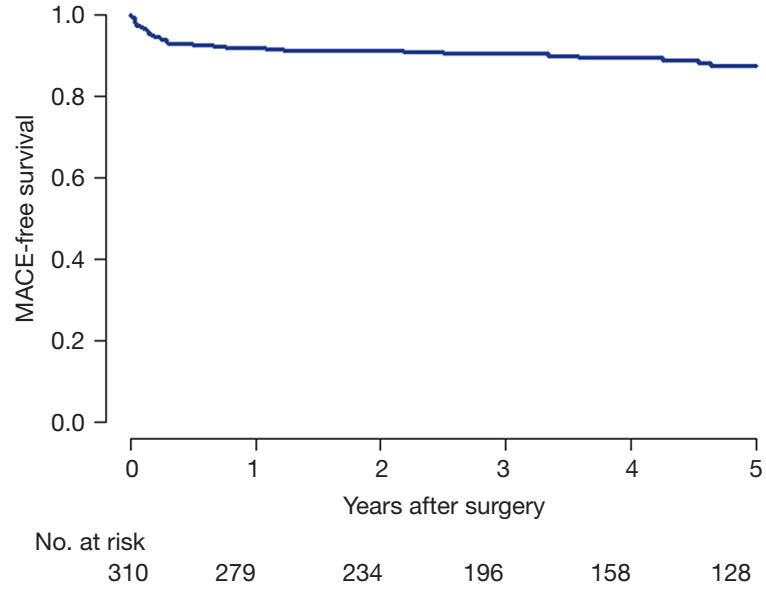

Figure 1 Kaplan-Meier curve for MACE-free survival. MACE, major adverse cardiac events.

\begin{tabular}{ll}
$\begin{array}{l}\text { Table } 4 \text { The last follow-up echocardiographic profiles }(>6 \text { months } \\
\text { after MV repair) ( } \mathrm{n}=295)\end{array}$ & $\mathrm{n}=295$ \\
\hline Echocardiographic data & $60.4 \pm 6.4$ \\
\hline LV ejection fraction, \% & $32.0 \pm 5.4$ \\
LV end-systolic dimension, $\mathrm{mm}$ & $49.4 \pm 5.2$ \\
LV end-diastolic dimension, $\mathrm{mm}$ & $40.2 \pm 7.4$ \\
LA diameter, mm & $22.1 \pm 6.0$ \\
Peak TR pressure gradient, $\mathrm{mmHg}$ & $2(0.6)$ \\
TR $\geq$ grade 3 & \\
MR grade & $139(47.1)$ \\
None or trace & $84(28.5)$ \\
1 & $40(13.6)$ \\
2 & $24(8.1)$ \\
3 & $8(2.7)$ \\
4
\end{tabular}

Values are $\mathrm{n}(\%)$, or mean \pm standard deviation, unless otherwise indicated. LV, left ventricular; LA, left atrium; TR, tricuspid regurgitation; MR, mitral regurgitation.

stroke in four (1.3\%) patients, infective endocarditis in two $(0.6 \%)$, and reoperation in two $(0.6 \%)$. MACE-free survival at 5 years was $87.6 \%$ (Figure 1 ).

\section{Echocardiographic results}

Late postoperative echocardiographic profiles ( $>6$ months

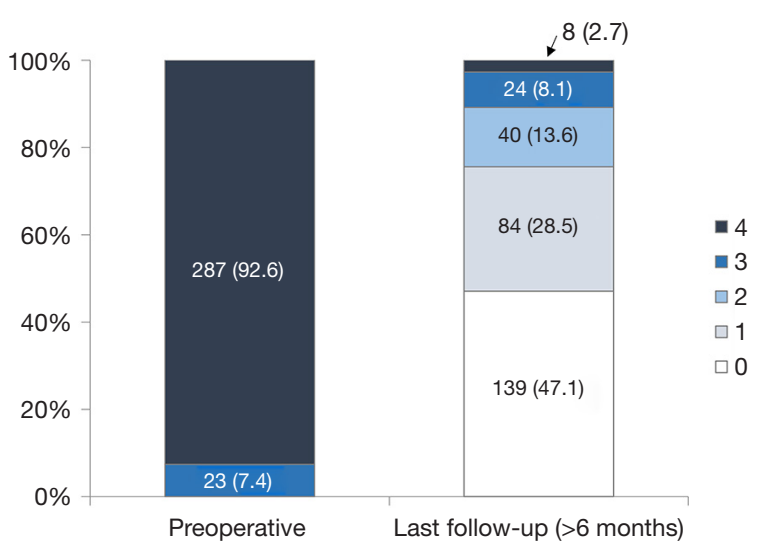

Figure $2 \mathrm{MR}$ results at the preoperative and the last follow-up echocardiographic assessment. MR, mitral regurgitation.

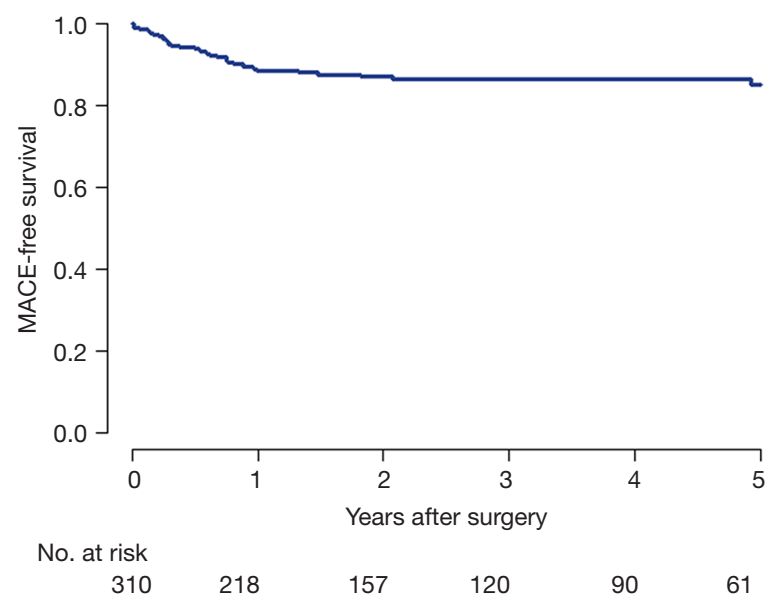

Figure 3 Kaplan-Meier curve for freedom from moderate MR. MR, mitral regurgitation.

after MV repair) were obtained in 295 of 310 (95.2\%) patients. The median follow-up duration was 35.2 months (IQR, 18.0 to 61.9 months). During follow-up, 32 (10.8\%) patients developed MR $\geq$ grade 3 (Table 4, Figure 2). Freedom from significant MR (> grade 2$)$ at 5 and 8 years was $86.5 \%$ and $82.9 \%$, respectively (Figure 3 ).

\section{Procedural time in chronological case order}

Both ACC and CPB time tended to decrease by the chronological order of MVP cases (Figure 4). The results of the Pearson correlation analysis for each procedural time were as follows: ACC time $(\mathrm{r}=-0.346, \mathrm{P}<0.001)$, CPB time $(\mathrm{r}=-0.185, \mathrm{P}=0.001)$. The Pearson analysis demonstrated 
A

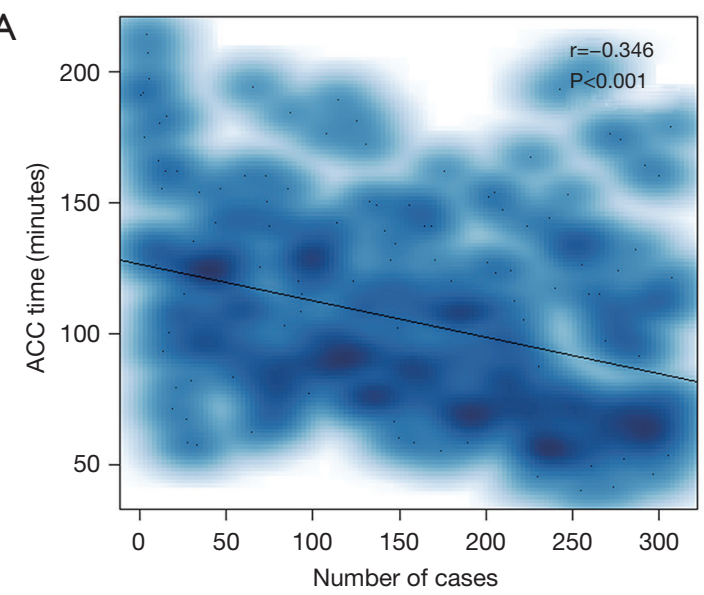

B

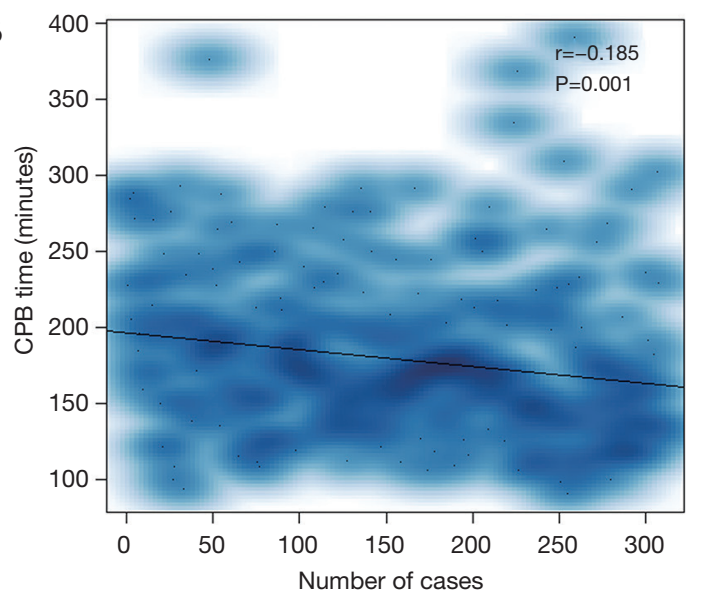

Figure 4 Scatterplots with slopes for changes in (A) aortic cross-clamping (ACC) time and (B) cardiopulmonary bypass (CPB) time by the chronological order of mitral valve repair. $r$, Pearson coefficient.

that both ACC and CPB time were correlated with surgical experience, manifested by chronological case order.

\section{Discussion}

Our institution has performed MICS (Minimally Invasive Cardiac Surgery) via right mini-thoracotomy using the AESOP 3000 [system] (Computer Motion, Santa Barbara, California, USA) (7) since 2002, preceding the introduction of robotic MV repair in 2007. The use of minimally invasive approach has steadily grown to be a mainstay of surgical methods in our institution, as the clinical outcomes of MV repair are reported to be comparable to conventional sternotomy $(3,7,8)$. A myriad of experiences in minimally invasive $M V$ repair via right mini-thoracotomy were the basis for successful establishment and favorable clinical results of robotic MV repair.

Robotic MV repair using da Vinci Surgical System shares similarities with other approaches via right minithoracotomy. These similarities include the use of peripheral cannulation techniques for instituting cardiopulmonary bypass (CPB) and approaching the MV anatomy from the right side of the chest (1). In addition to the well-known clinical advantages of MICS such as alleviated postoperative pain and fast recovery, use of the da Vinci Surgical System may enhance MICS performance. Such advancements are achieved by providing high-definition, three-dimensional visualization of the surgical field and sophisticated, tremorfree movement of the robotic instruments. Despite the potential advantages of robotic MV repair, the use of the da
Vinci Surgical System has been relatively stagnant in Asian countries because of concerns regarding its safety, efficacy, durability, and high cost.

Since the beginning of robotic MV repair, the possibility of increased surgical risks associated with the use of telemanipulation robotic instruments in a closed chest cavity has been frequently critiqued $(9,10)$. In particular, use of robotic instruments which lack tactile feedback may not be suited to complex surgical MV repairs. In addition, stroke risk may increase due to the routine use of retrograde systemic perfusion techniques in the setting of MICS. However, the clinical outcomes of robotic MV repair performed by experienced robotic surgeons in highvolume institutions have proved to be comparable to the outcomes by conventional sternotomy or right thoracotomy approaches $(3,5,10)$. Seco et al. (5) reported the outcomes of robotic MV surgery as satisfactory, with early mortality less than $1.0 \%$ and low rates of early major morbidity (myocardial infarction $0.0-3.2 \%$, stroke $0.0-0.3 \%$, bleeding $0.0-0.3 \%$ and wound problems $1.1-6.0 \%$ ).

The present study also emulates the excellent clinical outcomes of the previous studies. Our institution demonstrated no early mortality and a low incidence of early complications (CVA 1.0\%, bleeding $3.5 \%$ and reoperation $1.0 \%)$. The rate of postoperative bleeding $(\mathrm{n}=11,3.5 \%)$ is relatively high in this study. Most bleeding originated from the intercostal muscles at port entry sites. Our institution has not had postoperative bleeding since August 2013 as our assistant surgeons become more experienced in exploring the pleural cavity before wound closure. Stroke and aortic 
root injury leading to aortic dissection can be catastrophic. Since the beginning of robotic MICS program, we have routinely screened for aorto-iliac atherosclerotic disease using computed tomography (CT) and adjusted cannulation strategies accordingly. There have been no cases of early stroke since January 2013. However, among the concerns for adapting robotic $M V$ repair, safety is the most essential aspect; unless the robotic MV repair can attain the same level of safety as conventional MV repair does, efficacy and durability of robotic $M V$ repair are not even worth the consideration. Specifically, in regards to safety, aortic root injury resulting in iatrogenic aortic dissection must be avoided. One of the many requisites to minimize root injury, as well as other complications, is a bedside assistant surgeon that is proficient in many integral handheld surgical tasks. Thus, the existence of dedicated bedside assistant surgeons is indispensable in maintaining the excellent outcomes of robotic MV repair.

In the present study, we reported 305 (98.4\%) of 310 patients who underwent successful robotic $M V$ repairs in the immediate postoperative period. Among five patients with early failure of MV repair, two patients required early reoperation within 30 days and one patient underwent late reoperation after 5 years. Freedom from significant MR (> grade 2) at 5 and 8 years was $86.5 \%$ and $82.9 \%$, respectively. In our previous study, we reported 5 years freedom from significant MR as $86.1 \%$ and $85.3 \%$ after MV repair via right mini-thoracotomy and conventional sternotomy, respectively (7). These results suggest that robotic MV repair offers excellent long-term durability with minimal surgical risks compared to conventional or other minimally-invasive approaches.

The pathological involvement of the anterior MV leaflet is considered to be difficult to repair, and is therefore prone to late recurrence of MR (11-13). The motion to use robotic methods to repair the anterior leaflet has remained controversial, since this procedure requires sophisticated techniques. In our study, 132 (45.8\%) patients had involvement of the anterior leaflet but underwent successful MV repair using various repair techniques (Table 2). Likewise, Rodriguez et al. reported their studies of 66 patients with anterior leaflet or bileaflet prolapse and demonstrated that robotic MV repair for these prolapses is feasible without device-related complications (13). Suri et al. also asserted that robotic MV repair can allow for complete correction of all subsets of leaflet prolapse (12). In accordance with these mentioned studies, our results confirm that complex MV prolapse can be safely and efficaciously repaired using robotic techniques.

Moreover, other concomitant valvular pathologies can be addressed through robotic techniques $(14,15)$. We performed the Maze procedure and tricuspid annuloplasty concurrent with MV repair in $65(20.9 \%)$ and $43(13.8 \%)$ patients, respectively. Additionally, Nifong et al. reported their findings of $86(15.9 \%)$ preoperative atrial fibrillation (AF) patients which demonstrated $96.5 \%$ of freedom from AF despite long operative times (14). In our study, combining these procedures with MV repair did not significantly increase operative adverse events. Therefore, the consolidation of these results as well as the expected advancement of robotic technology in the coming decades suggest that simultaneous cardiac procedures during robotic MV repair may be more prevalent.

In conclusion, as made clear by the results discussed in this study, robotic MV repair can be safely and efficaciously performed with excellent short- and long-term clinical outcomes. The durability of robotic MV repair is similar to those by conventional sternotomy or right minithoracotomy approaches. Furthermore, complex MV repair techniques as well as concomitant cardiac procedure such as $\mathrm{AF}$ ablation or tricuspid repair can be addressed through robotic techniques.

\section{Acknowledgements}

None.

\section{Footnote}

Conflicts of Interest: The authors have no conflicts of interest to declare.

\section{References}

1. Suri RM, Dearani JA, Mihaljevic T, et al. Mitral valve repair using robotic technology: Safe, effective, and durable. J Thorac Cardiovasc Surg 2016;151:1450-4.

2. Bush B, Nifong LW, Alwair H, et al. Robotic mitral valve surgery-current status and future directions. Ann Cardiothorac Surg 2013;2:814-7.

3. Mihaljevic T, Koprivanac M, Kelava M, et al. Value of robotically assisted surgery for mitral valve disease. JAMA Surg 2014;149:679-86.

4. Paul S, Isaacs AJ, Jalbert J, et al. A population-based analysis of robotic-assisted mitral valve repair. Ann Thorac Surg 2015;99:1546-53. 
5. Seco M, Cao C, Modi P, et al. Systematic review of robotic minimally invasive mitral valve surgery. Ann Cardiothorac Surg 2013;2:704-16.

6. Yoo JS, Kim JB, Jung SH, et al. Mitral durability after robotic mitral valve repair: analysis of 200 consecutive mitral regurgitation repairs. J Thorac Cardiovasc Surg 2014;148:2773-9.

7. Yoo JS, Kim JB, Jung SH, et al. Echocardiographic assessment of mitral durability in the late period following mitral valve repair: minithoracotomy versus conventional sternotomy. J Thorac Cardiovasc Surg 2014;147:1547-52.

8. Glauber M, Miceli A. State of the art for approaching the mitral valve: sternotomy, minimally invasive or total endoscopic robotic? Eur J Cardiothorac Surg 2015;48:639-41.

9. Murphy DA, Moss E, Binongo J, et al. The Expanding Role of Endoscopic Robotics in Mitral Valve Surgery: 1,257 Consecutive Procedures. Ann Thorac Surg 2015;100:1675-81; discussion 1681-2.

10. Ramzy D, Trento A, Cheng W, et al. Three hundred robotic-assisted mitral valve repairs: the Cedars-Sinai experience. J Thorac Cardiovasc Surg 2014;147:228-35.

Cite this article as: Kim HJ, Kim JB, Jung SH, Lee JW. Clinical outcomes of robotic mitral valve repair: a single-center experience in Korea. Ann Cardiothorac Surg 2017;6(1):9-16. doi: $10.21037 /$ acs.2016.10.02
11. Nishimura RA, Otto CM, Bonow RO, et al. 2014 AHA/ ACC guideline for the management of patients with valvular heart disease: a report of the American College of Cardiology/American Heart Association Task Force on Practice Guidelines. J Thorac Cardiovasc Surg 2014;148:e1-e132.

12. Suri RM, Burkhart HM, Daly RC, et al. Robotic mitral valve repair for all prolapse subsets using techniques identical to open valvuloplasty: establishing the benchmark against which percutaneous interventions should be judged. J Thorac Cardiovasc Surg 2011;142:970-9.

13. Rodriguez E, Nifong LW, Chu MW, et al. Robotic mitral valve repair for anterior leaflet and bileaflet prolapse. Ann Thorac Surg 2008;85:438-44; discussion 444.

14. Nifong LW, Rodriguez E, Chitwood WR Jr. 540 consecutive robotic mitral valve repairs including concomitant atrial fibrillation cryoablation. Ann Thorac Surg 2012;94:38-42; discussion 43.

15. Lewis CT, Stephens RL, Tyndal CM, et al. Concomitant robotic mitral and tricuspid valve repair: technique and early experience. Ann Thorac Surg 2014;97:782-7. 